\title{
Treatment Outcomes of Epithelial Ovarian Cancers Following Maximum Cytoreduction and Adjuvant Paclitaxel-Carboplatin Chemotherapy: Egyptian NCI Experience
}

\author{
Hanan Ramadan Nassar ${ }^{1 *}$, Ahmed A Zeeneldin ${ }^{1}$, Amany Mohamed Helal ${ }^{1}$, Yahia \\ Mahmoud Ismail ${ }^{1}$, Abeer Mohamed Elsayed ${ }^{2}$, Mohamed A Elbassuiony ${ }^{1}$, Manar \\ M Moneer ${ }^{3}$
}

\begin{abstract}
Background: Epithelial ovarian cancer (EOC) is the commonest malignancy involving the ovaries. Maximum surgical cytoreduction (MCR) followed by adjuvant taxane-platinum chemotherapy are the standard of care treatments. Aims: To study treatment outcomes of EOC patients that were maximally cyto-reduced and received adjuvant paclitaxel-carboplatin (PC) chemotherapy. Materials and Methods: This retrospective cohort study included 174 patients with EOC treated at the Egyptian National Cancer Institute between 2006 and 2010. For inclusion, they should have had undergone MCR with no-gross residual followed by adjuvant PC chemotherapy. MCR was total abdominal hysterectomy/bilateral salpingo-oophorectomy [TAH/BSO] or unilateral salpingooophorectomy [USO] plus comprehensive staging. Results: The median age was 50 years. Most patients were married $(97.1 \%)$, had offspring $(\mathbf{9 2 . 5 \%})$, were postmenopausal $(\mathbf{5 3 . 4 \%})$, presented with abdominal/pelvic pain and swelling $(93.7 \%)$, had tumors involving both ovaries $(45.4 \%)$ without extra-ovarian extension i.e. stage I $(55.2 \%)$ of serous histology $(79.9 \%)$ and grade II (87.4\%). TAH/BSO was performed in $97.7 \%$ of cases. A total of 1,014 PC chemotherapy cycles were administered and were generally tolerable with 93.7\% completing 6 cycles. Alopecia and numbness were the commonest adverse events. The median follow up period was 42 months. The 2-year rates for disease free survival (DFS) and overall survival (OS) were 70.7\% and 94.8\%, respectively. The respective 5-year rates were $52.6 \%$ and $81.3 \%$. Advanced stage and high-grade were significantly associated with poor DFS and $O S(p<0.001)$. Age $>65$ years was associated with poor $O S(p=0.008)$. Using Cox-regression, stage was independent predictor of poor DFS and OS. Age was an independent predictor of poor OS.
\end{abstract}

Keywords: Ovarian neoplasms - epithelial - Egypt, National Cancer Institute - cytoreduction - adjuvant chemotherapy

Asian Pac J Cancer Prev, 16 (16), 7237-7242

\section{Introduction}

Epithelial OC (EOC) is the commonest among different malignant ovarian tumors. EOCs are believed to arise from the surface epithelium of the ovary. However, some evidence suggests that Fallopian tube could also be the source of some OCs (Piek et al., 2008). Worldwide, ovarian cancer (OC) is the 6th most common cancer in females and the 2nd gynecologic cancer. It is the 8th most common cause of cancer mortality in females and the 2nd lethal gynecologic cancer. These figures differ by region. In developed regions incidence and mortality of OC exceeds that of cervix uteri. In developing regions, the situation is reversed (Garcia et al., 2007). EOC is the leading cause of mortality from gynecologic cancer in the United States and is the fifth most common cause of cancer mortality in women with a less than $40 \%$ cure rate (Siegel et al., 2014).The incidence of OC increases with age and is most prevalent in the sixth and seventh decades of life.

In the Arab world, the frequency of OC varies from one country to the other, it accounts for $6.3 \%$ in Oman, $5.7 \%$ in Bahrain, $5.1 \%$ in Jordan, $4.6 \%$ in Somalia, $4.2 \%$ in Algeria, $4 \%$ in Egypt, $3.6 \%$ in Saudi Arabia, $3.5 \%$ in Qatar, 3.4\% in Tunisia, 3.1\% in Kuwait, 2.8\% in Lebanon and $1.9 \%$ in UAE (El-Attar I., 2005). It is the 6th cause of cancer death in Egyptian females (Ferlay et al., 2012). At the Egyptian National Cancer Institute (NCI-Egypt), $\mathrm{OC}$ is the 5 th among females constituting $3.8 \%$ of their total and the most common gynecologic cancer (Alieldin., 2014). In the pathology department of NCI-Egypt, tumors of the female genital system represented $4.7 \%$ of total malignancy and OCs represented 29.2\% of them (Mokhtar et al., 2007).

Surgery is the main line of treatment in most EOCs and adjuvant chemotherapy is recommended for patients with stage IC and up and high grade tumors (Vergote et al., 
Hanan Ramadan Nassar et al

2010). The prognosis of early -stage disease is significantly better than late stage disease. The 5-year survival in early stages ranges between $80 \%$ and $93 \%$, while in late stages it is less than $30 \%$ (Heintz, 2006). Trials confirmed the survival benefit of immediate adjuvant chemotherapy in early stage EOCs (Colombo et al., 2003; Trimbos et al., 2003). The primary analysis of ICON1 trial showed a significant improvement in both recurrence free survival and overall survival (Trimbos et al., 2003). Very similar finding were reported in the ACTION trial (Colombo et al., 2003).

Despite $\mathrm{OC}$ is the commonest female malignancy at NCI-Egypt, its treatment outcomes were not previously reported. The objective of this study is to report the treatment outcomes of EOC patients that were maximally cyto-reduced and received adjuvant paclitaxel-carboplatin (PC) chemotherapy.

\section{Materials and Methods}

This is a retrospective cohort study that included patients with a confirmed histologic diagnosis of EOC $(\mathrm{n}=$ 174) presenting to NCI- Egypt between the years 2006 and 2010 that were treated with maximally cytoreduction followed by post-operative (adjuvant) paclitaxelcarboplatin (PC) chemotherapy. Maximum cyctoreduction was judged on the operative report that stated there is no visible residual after surgery and on the post-operative CT scan of the abdomen and pelvis that showed no evidence of residual disease.

Paclitaxel and carboplatin were administered on day 1 of every 21-days cycle for a maximum of 6 cycles. Paclitaxel was administered as 3-hour IV infusion in a dose of $175 \mathrm{mg} / \mathrm{square}$ meter. Carboplatin was administered as 1-hour IV infusion with area under the concentration curve (AUC) of 5-6. Patients were followed up till October 2012.

The study was approved by the Institutional Review Board of NCI-Egypt. Overall survival (OS) is calculated from the date of diagnosis till the date of death from any cause or last follow up. Disease-free survival (DFS) was calculated from the date of surgery till the date of relapse, death or last follows up.

\section{Statistical analyses}

These were done using IBM SPSS ${ }^{\circledR}$ version 21.0. Nominal and categorical variables were compared using the Chi-squared or Fisher-Exact tests. Numerical variables were compared using t-test or Man-Whitney's test. Unadjusted survival was estimated using the KaplanMeier method and groups were compared using the logrank test. Step-wise Cox regression Hazards Model was used to calculate adjusted survival for each treatment, controlling for patients covariates. A probability $(p) \leq 0.05$ was considered statistically significant. The primary endpoint was OS. Secondary endpoints included DFS and treatment-related toxicities.

\section{Results}

\section{Patients' characteristics}

This single-center retrospective cohort study included
174 female patients with EOC who were maximally cytoreduced and received adjuvant paclitaxel-carboplatin chemotherapy combination. The median age was 50 years (range, 20-70 years). The majority of patients $(97.1 \%)$ were married and most $(92.5 \%)$ had offspring's (range, 1-9; median of 4). Postmenopausal status was encountered in $53.4 \%$ and positive family history was encountered in $5.2 \%$ (Table 1). Most patients presented by a combination of abdominal/pelvic pain and swelling. Most patients had tumors involving both ovaries without extra-ovarian extension i.e. stage I. Serous cyst-adenocarcinoma was the commonest histology and the tumor grade II predominated. Elevated CA125 tumor marker was encountered in $92 \%$ of patients with a median of $400 \mathrm{IU} / \mathrm{Ml}$ (range, 1-19860).

\section{Treatments}

TAH/BSO plus comprehensive staging was the classic procedure applied in $97.7 \%$ of cases. Unilateral ovariectomy plus comprehensive staging was applied in

Table 1. Characteristics of 174 Patients with EOC Treated at ENCI

\begin{tabular}{|c|c|c|}
\hline & $\begin{array}{c}\text { Number } \\
174\end{array}$ & $\begin{array}{c}\text { Percentage } \\
100\end{array}$ \\
\hline \multicolumn{3}{|l|}{ Menopausal status } \\
\hline Premenopausal & 81 & 46.6 \\
\hline Postmenopausal & 93 & 53.4 \\
\hline \multicolumn{3}{|l|}{ Marital status } \\
\hline Single & 5 & 2.9 \\
\hline Married & 169 & 97.1 \\
\hline \multicolumn{3}{|l|}{ Family history } \\
\hline Negative & 165 & 94.8 \\
\hline Positive & 9 & 5.2 \\
\hline \multicolumn{3}{|l|}{ Complaint } \\
\hline Mass & 2 & 1.1 \\
\hline Pain & 9 & 5.2 \\
\hline Mixed & 163 & 93.7 \\
\hline \multicolumn{3}{|l|}{ Laterality } \\
\hline Right & 61 & 35.1 \\
\hline Left & 34 & 19.5 \\
\hline Bilateral & 79 & 45.4 \\
\hline \multicolumn{3}{|l|}{ Stage } \\
\hline I & 96 & 55.2 \\
\hline II & 64 & 36.8 \\
\hline III & 10 & 5.7 \\
\hline $\mathrm{IV}^{*}$ & 4 & 2.3 \\
\hline \multicolumn{3}{|l|}{ Pathology } \\
\hline Serous & 139 & 79.9 \\
\hline Endometroid & 21 & 12.1 \\
\hline Mucinous & 9 & 5.2 \\
\hline Clear Cell & 1 & 0.6 \\
\hline Others & 4 & 2.3 \\
\hline \multicolumn{3}{|l|}{ Grade } \\
\hline I & 1 & 0.6 \\
\hline II & 152 & 87.4 \\
\hline III & 21 & 12.1 \\
\hline \multicolumn{3}{|l|}{ Surgery } \\
\hline $\mathrm{TAH} / \mathrm{BSO}+\mathrm{CS}$ & 170 & 97.7 \\
\hline $\mathrm{USO}+\mathrm{CS}$ & 4 & 2.3 \\
\hline
\end{tabular}

*EOC: epithelial ovarian cancer, ENCI: Egyptian National Cancer Institute, TAH/BSO: total abdominal hysterectomy and bilateral salpingo-oophorectomy, CS: comprehensive staging, USO: unilateral salpingo-oophorectomy 
4 patients $(2.3 \%)$ aged $20-28$ years and all had stage IA grade II tumors. The 174 patients received 1014 cycles of paclitaxel-carboplatin chemotherapy. The median number of cycles per patient was 6 cycles (range, 2-6 cycles).

Treatment was generally tolerable with only 5 patients $(2.9 \%)$ not completing the planned number of cycles. Dose reduction was applied in six patients due to repeated neutropenia. Alopecia was the commonest adverse event (AE) and was encountered in $98.3 \%$ (Table 2).

\section{Survival}

After a median follow-up period of 42 months $(95 \%$ CI, 38.7-45.2), 69 patients developed recurrence and 25 patients were dead.All of the dead patients had a preceding recurrence. The median overall survival was not reached. The 2-year OS rate \pm standard error (SE) was $94.8 \% \pm 1.7 \%$ and the 5-year OS rate was $81.3 \% \pm 3.8 \%$ (Figure 1). Compared to the other counterparts, OS was significantly longer in patients who were non-elderly (i.e. $<65$ years),

Table 2. Adverse events in 174 patients with EOC receiving adjuvant $\mathrm{PC}$ Chemotherapy

\begin{tabular}{lc}
\hline Toxicity & All \\
\hline Premature stoppage & $5(2.8 \%)$ \\
Alopecia & $171(98.3 \%)$ \\
Neurotoxicity & $105(60 \%)$ \\
Neutropenia & $73(42 \%)$ \\
Anemia & $45(26 \%)$ \\
Diarrhea & $78(45 \%)$ \\
Thrombocytopenia & $99(57 \%)$ \\
Nausea ,vomiting & $105(60 \%)$ \\
\hline
\end{tabular}

*EOC: epithelial ovarian cancer, PC: paclitaxel-carboplatin

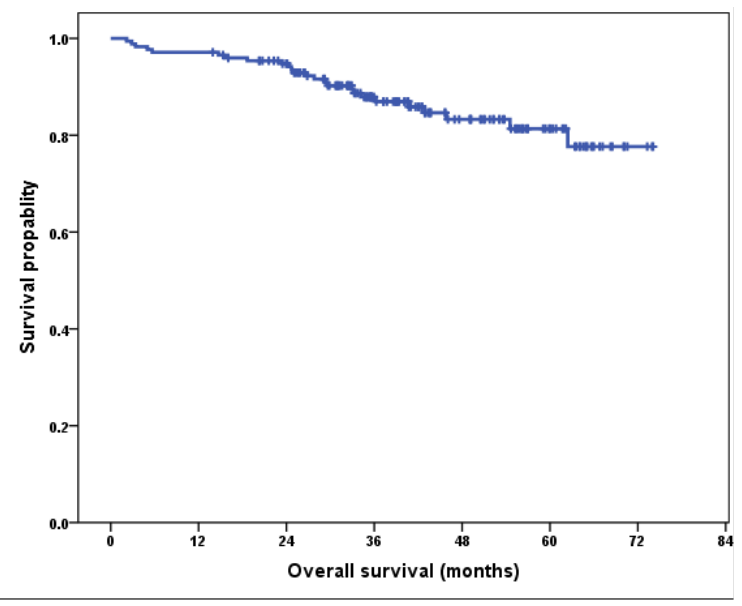

Figure 1. Overall Survival in 174 Patients with Epithelial Ovarian Cancer

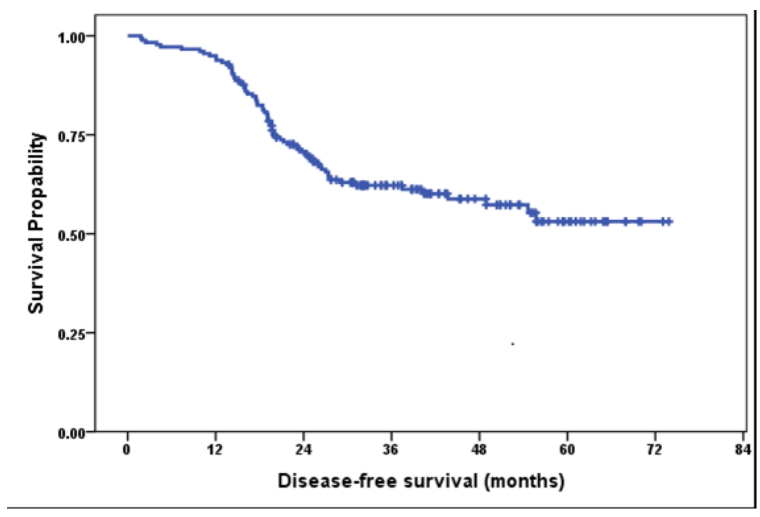

Figure 2.Disease-free Survival in 174 Patients with Epithelial Ovarian Cancer

Table 3. Factors Impacting OS and DFS in 174 Patients with EOC

\begin{tabular}{|c|c|c|c|c|c|c|c|}
\hline \multirow[b]{2}{*}{ Factor } & \multirow[b]{2}{*}{$\mathrm{N}$} & \multicolumn{3}{|c|}{ DFS } & \multicolumn{3}{|c|}{ OS } \\
\hline & & 2YR (SE) & 5YR (SE) & $\mathrm{P}$ & 2YR (SE) & 5YR (SE) & $\mathrm{P}$ \\
\hline All & 174 & $70.7(3.5)$ & $52.6(4.9)$ & -- & $94.8(1.7)$ & $81.3(3.8)$ & -- \\
\hline \multicolumn{8}{|l|}{ Age } \\
\hline$\leq 65 y$ & 169 & $71.0(3.5)$ & $52.7(5.0)$ & & $95.8(1.5)$ & $82.2(3.8)$ & \\
\hline$>65 y$ & 5 & $60.0(21.9)$ & $60.0(21.9)$ & 0.741 & $60.0(21.9)$ & $60.0(21.9)$ & 0.008 \\
\hline \multicolumn{8}{|l|}{ Menopausal status } \\
\hline Premenopausal & 81 & $80.7(4.5)$ & $55.6(7.7)$ & & $97.5(1.7)$ & $86.5(5.0)$ & \\
\hline Postmenopausal & 93 & $62.1(5.1)$ & $51.2(5.8)$ & 0.057 & $92.4(2.7)$ & $76.4(5.9)$ & 0.071 \\
\hline \multicolumn{8}{|l|}{ CA125 } \\
\hline$\leq 35 \mathrm{IU} / \mathrm{Ml}$ & 13 & $83.9(7.4)$ & $71.9(14.3)$ & & $100.0(0)$ & $83.3(15.2)$ & \\
\hline$>35 \mathrm{IU} / \mathrm{Ml}$ & 161 & $69.7(3.7)$ & $51.3(5.2)$ & 0.239 & $94.4(1.8)$ & $81.1(3.9)$ & 0.497 \\
\hline \multicolumn{8}{|l|}{ Pathology } \\
\hline Serous & 160 & $70.9(3.6)$ & $51.2(5.2)$ & & $96.2(1.5)$ & $81.7(4.0)$ & \\
\hline Others & 14 & $68.8(13.3)$ & $68.8(13.3)$ & 0.652 & $78.6(11.0)$ & $78.6(11.0)$ & 0.249 \\
\hline \multicolumn{8}{|l|}{ Stage } \\
\hline I & 96 & $84.4(3.7)$ & $68.0(6.2)$ & & $97.9(1.5)$ & $93.4(3.0)$ & \\
\hline II & 64 & $60.9(6.2)$ & $45.5(6.7)$ & & $93.6(3.1)$ & $81.1(5.5$ & \\
\hline III-IV & 14 & $24.5(12.1)$ & 0 & $<0.001$ & $78.6(11.0)$ & $26.9(13.1)$ & $<0.001$ \\
\hline \multicolumn{8}{|l|}{ Grade } \\
\hline I-II & 153 & $74.2(3.6)$ & $58.3(4.9)$ & & $96.0(1.4)$ & $88.3(2.9)$ & \\
\hline III & 21 & $41.9(11.7)$ & 0 & 0.001 & $85.4(7.8)$ & $34.9(14.9)$ & $<0.001$ \\
\hline \multicolumn{8}{|l|}{ Surgery } \\
\hline $\mathrm{TAH} / \mathrm{BSO}+\mathrm{CS}$ & 170 & $70.7(3.5)$ & $51.7(5.0)$ & & $94.7(1.7)$ & $81.0(3.9)$ & \\
\hline $\mathrm{USO}+\mathrm{CS}$ & 4 & $100.0(0.0)$ & $100.0(0.0)$ & 0.178 & $100.0(0.0)$ & $100.0(0.0)$ & 0.461 \\
\hline
\end{tabular}

OS: overall survival, DFS: disease free survival, TAH/BSO: total abdominal hysterectomy and bilateral salpingo-oophorectomy, CS: comprehensive staging, USO: unilateral salpingo-oophorectomy 
had non-advanced stage disease and low grade tumors (Table 3). Using multivariate analysis, age and stage were independent predictors of poor OS. Compared to stage I, the hazards of poor OS in stage II, III and IV was 4, 40 and 54 times ( $\mathrm{p}=0.011,0.010$ and 0.004 , respectively). Compared to patients ages $<65$ years, the hazards of poor OS in patients aged $>65$ years was 13 times $(p=0.001)$.

The median DFS was not reached. The 2-year DFS rate was $70.7 \% \pm 3.5 \%$. The 5-year DFS rate was $52.6 \% \pm 4.9 \%$ (Figure2). Compared to the other counterparts, OS was significantly longer in patients who had non-advanced stage disease and low grade tumors (Table 3). Using multivariate analysis, stage was an independent predictor of poor DFS state. Compared to stage I, the hazards of poor DFS in stage II, III and IV was 3, 22 and 15 times $(\mathrm{p}<0.001,0.001$ and 0.007 , respectively).

\section{Discussion}

Ovarian cancer is a common disease among females globally and in Egypt. It usually presents at an advanced stage and is a significant cause of morbidity and mortality. It is predominantly a disease of older, postmenopausal women with the majority of cases being diagnosed in women over 50 years (Alsop etal., 2012).

In the current study, the median age at diagnosis was 50 years with a range between 20 and 70 years. This is similar to prior Egyptian reports whether population-based or hospital-based or. In the Gharbiah population-based cancer registry, the median age at diagnosis was 49 years (Abd el-bar, 2007). The median age at Alexandria University hospitals was 48 years (Mohamed et al., 2012). This is similar to other developing countries like Pakistan where the median age at its National Cancer Institute was 49.5 years (Malik, 2002). US SEER data showed that the median age is in the late fifties and early sixties (Chan al., 2008; Fleming et al., 2009). The Middle East Cancer Consortium (MECC) evaluated the incidence of OC in Egypt, Israel, Cyprus and Jordan and compared it to the US SEER data base. This showed that the highest incidence in Cypriots, Israeli, and US SEER data were in the age group from 50 to 69, while in Egyptians, Jordanians, and Israeli Arabs, the highest age group was below the age of 50 years, which is very close to our results (Freedman et al., 2012). Thus, the median age in the current study is similar to other developing countries and almost 10 years lower than that in the developing world (Freedman et al., 2012). This finding is noticed in many other cancer types including breast cancer and may be related to differences in population structure and risk-factors (Zeeneldin et al., 2013).

Approximately 5\%-10\% of patients with OC develop the disease because of heredity with the breast and ovarian cancer syndrome due to mutations in the BRCA1 and BRCA2 genes and hereditary nonpolyposis colorectal cancer (HNPCC) being the major genetic causes (Domanska et al., 2007). In this study, positive family history was encountered in $5.2 \%$ of the patients. This is comparable to the lower end of the range in Western countries (5-10\%) (Wong and Auersperg, 2003) and lower than the 18\%-20\% figure from Pakistan
(Saeed and Akram, 2012, Malik, 2002). Further genetic and epidemiologic studies are needed to explain these results as underlying genetic mutations vary between communities (Fourati et al., 2014)

Among EOC patients of the current study, serous carcinoma predominated (79.9\%). This is similar to US, Jordan, Cyprus and some other countries (Paes et al., 2011; Freedman et al., 2012). However, the frequency is higher in the current study. However, adenocarcinoma NOS; 15 $20 \%$ in these reports; was not reported in our cases and this category may be added to the serous category thus its higher figure. Mucinous carcinoma in the current study (5.2\%) was comparable to US, Jordan and Cyprus (6-9\%) (Paes et al., 2011; Freedman et al., 2012) but lower than previous data from Egypt (16\%) (Freedman et al., 2012). Reasons for this are unknown. However, pathologists at NCI-Egypt are experienced and have many auxiliary techniques for adequate categorization of ovarian tumors.

Among patients in the current study; stages III and IV were seen in $8 \%$ of the cases. This contradicts with the general notion that $\mathrm{OC}$ is usually diagnosed at an advanced stage. At diagnosis, stage III and IV disease represent 55\%-80\% of EOCs (Malik, 2002; Sarwar et al., 2006; Paes et al., 2011). This discrepancy is related to the research question and hence the inclusion criteria of the current study that only included patients that are optimally cyto-reduced. Patients with early-stage disease are more likely to be optimally cyto-reduced than stage III and IV.

In this study CA-125 was elevated in more than $90 \%$ of all patients, while in the study done by Sarwar et al., CA-125 was elevated in only $70 \%$ of the cases of epithelial ovarian cancer (Sarwar et al., 2006) and Beth reported that it was elevated in about $80 \%$ his of cases especially late stage (Beth et al., 2005). About $45.5 \%$ of patients in the current study had bilateral tumors. This is close to the $35 \%$ previously reported from Gharbiah population-based cancer registry (Abd el-bar, 2007). It is higher than the figure from hospital-based frequencies. It is more than the $17.2 \%$ figure seen in an Asian study evaluating OC at a cancer hospital in a developing country (Beth et al., 2005 ) and also it is more than the $13 \%$ figure reported in Alexandria University hospital in Egypt (Mohamed, 2012).

Regarding the management, $97.7 \%$ of patients in the current study were treated with TAH/BSO plus complete staging and $2.3 \%$ underwent USO with complete staging. Singh et al., in the study evaluating OCs in oriental women from Singapore reported surgery as the primary treatment modality in $97 \%$ of the cases (Singh et al., 1990), while Sehouli et al., in a review of 372 consecutive patients with advanced OC in a university hospital in Berlin reported the use of surgery in $89 \%$ (Sehouli et al., 2010). Thrall et al., in their report evaluating patients with advanced EOC in the Medicare population found that surgery was performed initially in 58.8\% of the women (Thrall et al., 2011).

Adjuvant platinum-based chemotherapy is effective in prolonging the survival of majority of patients (Elit et al., 2004). The optimal adjuvant chemotherapy regimen and duration of treatment in early stage ovarian cancer is a subject of continuing research. The updated data from ICON1 trial confirmed the long-term recurrence- 
free survival and overall survival benefit from adjuvant platinum-based chemotherapy in women with early stage ovarian cancer (Collinson et al., 2014). Results are consistent with previous trials and meta-analysis (Colombo et al., 2003; Trimbos et al., 2010). The magnitude of benefit appears greatest in women with highrisk disease. Winter et al. (2012) reported that adjuvant platinum-based chemotherapy is effective in prolonging the survival of the majority of patients who are assessed as having early stage (FIGO stage I and II) EOC (WinterRoach et al., 2012). However it may be withheld from women in whom there is well-differentiated encapsulated unilateral disease. Others with unstaged early disease or those with poorly differentiated tumor should be offered chemotherapy. In this study all patients received adjuvant paclitaxel-carboplatin chemotherapy. The median number of cycles per patient was 6 cycles (range, 2-6 cycles). Alopecia was the commonest adverse event (AE) and was encountered in $98.3 \%$. Grade 3 and 4 toxicities were rare except 10 patients had grade 3 neutropenia. Neurotoxicity was encountered in $60 \%$ of patients without any grade 3 or 4 toxicity.

In the current study, the 5-year OS rate was $81.3 \%$. this is similar to the $87 \%$ Os rate reported by Bamias et al. (2006), the $85 \%$ OS rate of reported by Trimbos et al. (2003) and the 79\% figure reported by Colombo, (2003). We reported a 5-year DFS rate of 52.6\%. This is lower than the $73 \%-79 \%$ figures reported by other authors (Colombo et al., 2003; Bamias et al., 2006). The high rate of early censoring may have explained the low DFS rates in the current study. Relative resistance to chemotherapy may be another reason. Further studies are needed in this regard.

We reported that survival was higher in premenopausal than postmenopausal women $(86.5 \%$ vs. $76.4 \%)$. Similarly Chan et al. (2008) reported that women younger than 50 years old had superior 3-years OS than those aged 50 years or more $(93.1 \% v s .84 .2 \%, \mathrm{p}=0.001)$. Surgical staging and tumor grade are the prognostic factors identified in many studies (Colombo et al., 2003; Trimbos et al., 2003; Chan et al., 2008). In the current study, OS was significantly longer in patients who had non-advanced stage disease and low grade tumors. In Bamias study reported that "the combination of tumor stage and grade identified a subgroup (stage Ic or II and grade 2 or 3), which had a significantly inferior 5-year recurrence free survival compared with patients with only one of these factors (73\% vs 92\%) (Bamias et al., 2006).

In conclusion, the age incidence of EOC patients in the current study is ten years younger than that reported in US SEER data and other western countries. Papillary serous cyst-adenocarcinoma is the most predominant histological type. Most of ovarian patients in this study were presented with stage I, II. Surgical intervention is the main line of treatment, 6 cycles of paclitaxel-carboplatin is the most frequent chemotherapy regimen used. Age and stage were independent predictors of poor OS. Also stage was an independent predictor of poor DFS state.

\section{References}

Abd el-bar I (2007). Cancer in egypt, gharbiah. triennial report of 2000-2002, Gharbiah population based cancer registry. $1^{\text {st }}$ ed.; ISBN, 977-17-4932-3.

Alieldin N (2014). NCI Hospital based registry 2002-2010 [online].

Alsop K, Fereday S, Meldrum C, et al (2012). BRCA mutation frequency and patterns of treatment response in BRCA mutation-positive women with ovarian cancer, are ports from the australian ovarian cancer study group. J Clin Oncol, 30, 2654-63.

Bamias A, Papaddimitriou C, Efstathiou E, et al (2006). Four cycles of paclitaxel and carboplatin as adjuvant treatment in early-stage ovarian cancer, a six-year experience of the Hellenic Cooperative Oncology Group.BMC Cancer, 6, 228.

BethYK, Maurie AM, Patricia JE (2005). Ovarian cancer, fallopian tube carcinoma, and peritoneal carcinoma, in DeVita VT, Hellman S, Rosenberg SA (eds), Cancer, principles and practice of oncology. Philadelphia, PA, Lippincott Williams \& Wilkins.

Chan J, Fuh K, Shin J, Cheung M, Powell C, et al (2008). The treatment and outcomes of early-stage epithelial ovarian cancer, have we made any progress? Br J Cancer, 98, 1191-6.

Collinson F, QianW, Fossati R, et al (2014). Optimal treatment of early-stage ovarian cancer. Ann Oncol, 25, 1165-71.

Colombo N, Guthrie D, Chiari S, et al (2003). International collaborative ovarian neoplasm trial 1 , a randomized trial of adjuvant chemotherapy in women with early-stage ovarian cancer. J Natl Cancer Inst, 95, 125-32.

Domanska K, Malander S, Masback A, Nilbert M (2007). Ovarian cancer at young age, the contribution of mismatchrepair defects in a population-based series of epithelial ovarian cancer before age 40. Int J Gynecol Cancer, 17, 789-93.

El-Attar I (2005). Cancer in Arab world, magnitude of the problem UICC, 35-36.

Elit L, Chambers A, Fyles A, et al (2004). Systematic review of adjuvant cares for women with Stage I ovarian carcinoma. Cancer, 101, 1926-35.

Ferlay J, Soerjomataram I, Ervik M, et al (2013). GLOBOCAN 2012 v1 .0, cancer incidence and mortality worldwide, IARC Cancer Base No. 11 [Internet]. Lyon, France, International Agency for Research on Cancer

Fleming GF, Filiaci VL, Bentley RC (2009). Epithelial ovarian cancer. In RR barakat et al., eds., principles and practice of gynecologic oncology, $5^{\text {th }}$ ed., pp. 763-835. Philadelphia, Lippincott Williams and Wilkins.

Fourati A, Louchez MM, Fournier J, et al (2014). Screening for common mutations in BRCA1 and BRCA2 genes, interest in genetic testing of Tunisian families with breast and/or ovarian cancer. Bull Cancer, 101, 36-40.

Freedman LS, Al-Kayed S, Qasem MB, et al (2001). Cancer registration in the middle east. Epidemiol, 131-3.

Garcia M, Jemal A, Ward EM, et al (2007). Global cancer facts $\&$ figures. Atlanta GA, American Cancer Society.

Heintz AP, Odicino F, Maisonneuve P, et al (2006). Carcinoma of the ovary. FIGO $26^{\text {th }}$ annual report on the results of treatment in gynecological cancer. Int J Gynaecol Obstet, 95, 161-92.

Malik IA (2002). A prospective study of clinico-pathological features of epithelial ovarian cancer in Pakistan. J Pak Med Assoc, 52, 155-8.

Mohamed FM, Nour E, Noha A (2012). Retrospective analysis evaluating ovarian cancer cases presented at the clinical oncology department, alexandria university faculty of medicine. Alexandria J Med, 48, 353-60.

Mokhtar N, Gouda I, Adel I (2007). Cancer pathology registry 2003-2004and time trend analysis. Chapter 1; Table 1.4 ranking of some important malignant tumor, pathology department NCI. 


\section{Hanan Ramadan Nassar et al}

Paes MF, Daltoe RD, Madeira KP, et al (2011). A retrospective analysis of clinicopathologiocal and prognostic characteristics of ovarian tumors in the state espritio santo. Braz J Ovarian Res, 9, 4-14.

Piek JM, Van Diest PJ, Verheijen RH, et al (2008). Ovarian Carcinogenesis, an alternative hypothesis. Adv Exp Med Biol, 622, 79-87.

Saeed S, Akram M (2012). Epithelial ovarian cancer. epidemiology and clinicopathological features. Prof Med $J, 19,040-5$.

Sarwar CM, Siddiqui N, Khokhar RA, Badar F, et al (2006). Epithelial ovarian cancer at a cancer hospital in a developing country. Asian Pac J Cancer Prev, 7, 595-8.

Sehouli J, Savvatis K, Braicu E, et al (2010). Primary versus interval debulking surgery in advanced ovarian cancer, Results from a systematic single-center analysis. Int $J$ Gynecol Cancer, 20, 1331-40.

Siegel R, Ma J, Zou Z, Jemal A (2014). Cancer statistics. $C A$ Cancer J Clin, 64, 9-29.

Singh P, Arunachalam I, Singh P, et al (1990). Ovarian cancer in oriental women from singapore, disease pattern and survival. Int Surg, 75, 115-22.

Thrall MM, Gray HJ, Symons RG, et al (2011). Trends in treatment of advanced epithelial ovarian cancer in the Medicare population. Gynecol Oncol, 122, 100-6.

Trimbos JB, Parmar M, Vergote I, et al (2003), Two parallel randomized phase III trials of adjuvant chemotherapy in patients with early-stage ovarian carcinoma. european organization for research and treatment of cancer collaborators-adjuvant chemotherapy UN ovarian neoplasm. J Natl Cancer Inst, 95, 105-12.

Trimbos JB, Vergote I, Bolis G, et al (2003). Impact of adjuvant chemotherapy and surgical staging in early-stage ovarian carcinoma, european organisation for research and treatment of cancer adjuvant chemotherapy in ovarian neoplasm. J Natl Cancer Inst, 95, 113-25.

Trimbos B, Timmers P, Pecorelli S, et al (2010). Surgical staging and treatment of early ovarian cancer, long-term analysis from a randomized trial. J Natl Cancer Inst, 102, 982-87.

Vergote IB, Trope CG, Amant F, et al (2010). Neoadjuvant chemotherapy or primary surgery in stage IIIC or IV ovarian cancer. $N$ Engl J Med, 363, 943-53.

Winter-Roach BA, Kitchener HC, Dickinson HO, et al (2012). Adjuvant (post-surgery) chemotherapy for early stage epithelial ovarian cancer. Cochrane Database Syst Rev, 4706

Wong AS, Auersperg N (2003). Ovarian surface epithelium, family history and early events in ovarian cancer. Reprod Biol Endocrinol, 1, 70.

Zeeneldin AA, Ramadan M, Gaber AA, et al (2013). Clinicopathological features of breast carcinoma in elderly Egyptian patients, a comparison with the non-elderly using populationbased data. J Egypt Natl Canc Inst, 25, 5-11. 